\title{
(2) OPEN ACCESS \\ COVID-19: to be or not to be; that is the diagnostic question
}

\author{
Jamie J Coleman (D) , 1,2 Kaveh Manavi, ${ }^{2,3}$ Ella J Marson, ${ }^{1,2}$ Adam H Botkai, ${ }^{1,2}$ \\ Elizabeth Sapey ${ }^{2,3}$
}

${ }^{1}$ School of Medicine, University of Birmingham, Birmingham, UK ${ }^{2}$ Queen Elizabeth Hospital, University Hospitals Birmingham NHS Foundation Trust, Birmingham, UK

${ }^{3}$ Birmingham Acute Care Research Group, Institute of Inflammation and Ageing, University of Birmingham, Birmingham, UK

\section{Correspondence to} Professor Jamie J Coleman, Department of Clinical Pharmacology, University of Birmingham, Birmingham B15 2SP, West Midlands, UK: j.j.coleman@bham.ac.uk

Received 24 April 2020 Revised 28 April 2020 Accepted 29 April 2020 Published Online First 10 June 2020

\section{Check for updates}

(c) Author(s) (or their employer(s)) 2020. Re-use permitted under CC BY-NC. No commercial re-use. See rights and permissions. Published by BMJ.

To cite: Coleman JJ, Manavi

$\mathrm{K}$, Marson EJ, et al.

Postgrad Med J

2020:96:392-398

\section{ABSTRACT}

Since the first cases in December 2019, the severe acute respiratory syndrome coronavirus 2 (SARSCoV-2) has rapidly spread across the globe, resulting in the COVID-19 pandemic. Early clinical experiences have demonstrated the wide spectrum of SARS-CoV-2 presentations, including various reports of atypical presentations of COVID-19 and possible mimic conditions.

This article summarises the current evidence surrounding atypical presentations of COVID-19 including neurological, cardiovascular, gastrointestinal, otorhinolaryngology and geriatric features. A case from our hospital of pneumocystis pneumonia initially suspected to be COVID-19 forms the basis for a discussion surrounding mimic conditions of COVID-19. The dual-process model of clinical reasoning is used to analyse the thought processes used to make a diagnosis of COVID-19, including consideration of the variety of differential diagnoses.

While SARS-CoV-2 is likely to remain on the differential diagnostic list for a plethora of presentations for the foreseeable future, clinicians should be cautious of ignoring other potential diagnoses due to availability bias. An awareness of atypical presentations allows SARS-CoV-2 to be a differential so that it can be appropriately investigated. A knowledge of infectious mimics prevents COVID-19 from overshadowing other diagnoses, hence preventing delayed diagnosis or even misdiagnosis and consequent adverse outcomes for patients.

\section{INTRODUCTION}

In December 2019, the first patients presenting with a pneumonia of unknown origin were reported in the Wuhan province of the People's Republic of China. Over the subsequent months, the severe acute respiratory syndrome coronavirus 2 (SARS-CoV-2) has rapidly spread across the globe, resulting in the COVID-19 pandemic. As of 23 April 2020, there have been over 2.4 million confirmed cases worldwide in over 213 countries, areas or territories, resulting in 169000 deaths. ${ }^{1}$

Early clinical experiences in China demonstrated the wide spectrum of SARS-CoV-2 severity: from asymptomatic infection or mild upper respiratory tract illness, to severe viral pneumonia with respiratory failure and death. Patients with severe pneumonia are often hospitalised and a small proportion of these patients require mechanical ventilation and critical care support. ${ }^{2}$
The typical presentation of COVID-19 relates to the pathophysiology of the SARS-CoV-2 infection. SARS-CoV-2 is principally spread by aerosol transmission and is taken up into airway epithelial cells, and therefore the disease primarily affects the lungs. Research into SARS-CoV-2 has shown that in early stages of infection, only ciliated cells in the conducting airways are infected. In the $80 \%$ of patients with mild disease, only these conducting airways are affected, however $20 \%$ of patients will develop severe disease when the virus infects alveolar type II cells. ${ }^{3}$ Viral replication in these cells results in direct pathological insult to the lung parenchyma by causing inflammation and apoptosis. This damage typically results in pneumonia but can also progress to an acute respiratory distress syndrome (ARDS), which may lead to lifethreatening respiratory failure.

Viral replication throughout the body may lead to an excessive immune reaction or 'cytokine storm' due to haemophagocytic lymphohistiocytosis (HLH) in some patients. ${ }^{4}$ In most patients, there is an acute phase response which manifests as the systemic features of COVID-19, including fever, cytopenias and myalgias, and can result in multiple organ failure in a small proportion.

Like many infectious and inflammatory conditions, there are a myriad of clinical presentations resulting from SARS-CoV-2, hence many atypical features require a high level of clinical suspicion. This article discusses presentations of COVID-19 and presents some of the atypical presentations and mimic conditions to give an overview of the diagnostic nuances of this new virus.

\section{TYPICAL PRESENTATIONS OF COVID-19}

The case definition adopted by the UK has been developed using the typical presentation of COVID19. ${ }^{5}$ This is defined as patients who have required hospital admission and meet one of three clinical criteria (figure 1).

This case definition aligns with data from China outlining the most common presenting features of COVID-19 (table 1). ${ }^{6}$ Usual symptoms at onset of illness were fever, cough, myalgia or fatigue; whereas less common symptoms were headache, vomiting and diarrhoea.

\section{Diagnosis}

In patients with suspicious symptoms, confirmation of a COVID-19 diagnosis requires evidence of active SARS-CoV-2 infection. At present, laboratories require either an upper respiratory tract 


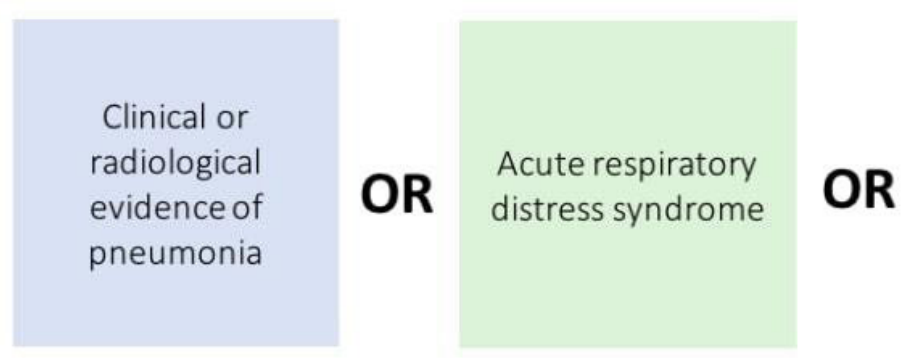

Figure 1 Case definition for COVID-19 in the UK. ${ }^{5}$

sample (nasopharyngeal and/or oropharyngeal swab) or a lower respiratory tract sample (sputum or tracheal aspirate) for testing. The presence of SARS-CoV-2 RNA is detected using reverse transcriptase PCR (RT-PCR). However, the performance of the biomolecular assay depends on both the stage of the disease and the site and operator accuracy of the sampling. When coupled with sampling errors, the overall sensitivity of the RT-PCR test is approximately $75 \% .^{7}$

\section{ATYPICAL PRESENTATIONS OF COVID-19}

Reports of atypical presentations of COVID-19 have been emerging as the SARS-CoV-2 pandemic has grown across the globe. Atypical presenting features refer to patients where the constitutional or respiratory symptoms are absent or minimal, and other non-respiratory symptoms or signs are early or predominant features.

\section{COVID-19 in the elderly: including delirium}

Studies have shown that the greatest risk factor for severe COVID-19 is increasing age, particularly patients with multimorbidity and/or higher frailty scores. ${ }^{3} 89$

Like many infections affecting elderly patients, COVID-19 can present as a non-specific decline. While most studies report elderly patients presenting with a fever and productive cough, this may be related to the case definition criteria being used to diagnose COVID-19. Elderly patients may in fact not develop the typical fever seen in SARS-CoV-2 infection due to a blunted response caused by immune senescence. ${ }^{10}$ 'Silent hypoxaemia', the phenomenon whereby patients develop hypoxaemia and respiratory failure without dyspnoea or respiratory distress, is another unusual presentation of COVID-19 that appears to particularly affect the elderly population. ${ }^{11}$ This is an especially worrying presentation as patients may feel well and therefore not present to hospital, and then subsequently precipitously

\begin{tabular}{ll}
\hline $\begin{array}{l}\text { Table } 1 \text { Common symptoms of SARS-CoV-2 } \\
\text { presentation }\end{array}$ & infection at \\
\hline Symptom & Patients (\%) \\
\hline Cough & 68 \\
\hline Fever & 44 \\
\hline Dyspnoea & 19 \\
\hline Myalgia & 15 \\
\hline Headache & 14 \\
\hline Vomiting & 5 \\
\hline Diarrhoea & 4 \\
\hline
\end{tabular}

SARS-CoV-2, severe acute respiratory syndrome coronavirus 2.
An influenza-like illness: Fever $>37.8^{\circ} \mathrm{C}$ and acute onset of $>1$ of the following respiratory symptoms:

- Persistent cough (with/without sputum)

- Hoarseness

- Nasal discharge or congestion

- Shortness of breath

- Sore throat

- Wheezing

- Sneezing

deteriorate due to their hypoxaemia, thus leading to treatment delay and adverse outcomes.

Delirium-the clinical manifestation of encephalopathy-is an important feature in COVID-19. Elderly patients infected with SARS-CoV-2 may present with delirium or subsequently develop delirium during the illness. However, the development of delirium is not exclusive to geriatric patients and may be seen in any patient with severe infection, particularly those on critical care. Delirium in its hyperactive motor form presents significant additional challenges in the context of management of admitted patients with COVID-19, due to increased risk of transmission to other patients and possible reduction in staffing levels. The British Geriatric Society recommends increased screening of high-risk groups (including patients with COVID-19) using the 4 'A's Test tool, and avoidance of common precipitants of delirium. $^{12}$

\section{Otorhinolaryngology features}

Smell and taste dysfunction has been reported in 34\%-59\% of patients with COVID-19. ${ }^{13}$ Anosmia (lack of the sense of smell) was an early observation in the pandemic and can present with or without taste disturbance (dysgeusia). It commonly arises early in the disease process or in patients with mild or no constitutional symptoms. ${ }^{14}$ The British Association of Otorhinolaryngology is now recommending anosmia and dysgeusia are added to the primary screening symptoms for COVID-19. ${ }^{15}$

Other rhinological symptoms typically seen in other respiratory viruses, such as rhinorrhoea or nasal congestion, are often absent in patients with COVID-19. ${ }^{14}$ Coronaviruses are known to be neurotropic, ${ }^{16}{ }^{17}$ and therefore isolated anosmia suggests SARS-CoV-2 invades the nervous system via the olfactory system specifically.

\section{Cardiovascular features}

Myocardial injury occurs early in SARS-CoV-2 infections, reflected in the rise of high-sensitivity troponin and natriuretic peptides, even in patients without known cardiovascular disease. ${ }^{18}$ However, it has been noted the pattern of rise of high-sensitivity troponin follows with other inflammatory markers (such as D-dimer, ferritin, lactate dehydrogenase), therefore this may be due to a 'cytokine storm' or secondary $\mathrm{HLH}$, rather than organic myocardial injury from the virus. ${ }^{19}$ A report produced by the National Health Commission of China shows that some patients with COVID-19 present with chest tightness and palpitations without fever and cough. ${ }^{18}$ This suggests the possibility of viral myocarditis or stress cardiomyopathy caused by the virus. Inflammation in the vascular system caused by SARS-CoV-2 can result in diffuse microangiopathy 
with microthrombosis. Likewise, inflammation in the myocardium can result in myocarditis, heart failure, cardiac arrhythmias, acute coronary syndrome, rapid deterioration and sudden death. ${ }^{20}$ Case reports from China showed mimics of ST-elevation myocardial infarctions without coronary obstruction in patients who are SARS-CoV-2 positive, with normalisation of ejection fraction and troponins as the patient recovered from COVID-19. ${ }^{21}$

Distinguishing the cause of these cardiac presentations as being due to SARS-CoV-2 or organic cardiovascular disease is difficult. Indeed, it is likely that SARS-CoV-2 both induces new cardiac pathologies and may exacerbate underlying cardiovascular diseases, ${ }^{22}$ in some cases leading to orthostatic hypotension and syncope. ${ }^{23}$

It remains imperative that appropriate treatment for heart failure, arrhythmias, acute coronary syndrome and thrombosis must be initiated regardless of the aetiology. However, clinicians should be aware of SARS-CoV-2 infection as a possible cause and investigate accordingly.

\section{Gastrointestinal and hepatic features}

Nausea and vomiting occur in approximately $5 \%$ of patients with COVID-19 and diarrhoea occurs in approximately $4 \% .{ }^{6}$ In early case series, including the first case of COVID-19 in the USA, ${ }^{24}$ gastrointestinal symptoms often predated any respiratory symptoms and, in some cases, have been the predominant feature of the illness. ${ }^{25}$ SARS-CoV-2 RNA has been isolated on anal swabs of patients with COVID-19, even after the virus has been cleared from the respiratory tract. ${ }^{26}$ This suggests that SARS-CoV-2 can replicate and cause symptoms in the gastrointestinal tract.

SARS-CoV-2 may cause liver injury, thus elevating liver aminotransferase enzyme levels in $16 \%-53 \%$ of patients with COVID-19. ${ }^{627-31}$ However, these results are usually discovered as a coexistent feature in more severe disease and not as the presenting feature. ${ }^{32}$ There are many potential causes for elevated aminotransferase levels including hypoxic hepatitis from respiratory compromise or drug-induced liver injury from current some of the COVID-19 treatments. Collateral liver damage may be caused by virally induced cytotoxic $\mathrm{T}$ cells, and the induction of a dysregulated innate immune response by the virus, explaining the association between deranged liver markers and COVID-19 severity. ${ }^{33}$ Therefore, clinicians should focus on treatment of the virus as this will improve the hepatic picture.

\section{Neurological features}

Preliminary studies report that approximately $40 \%$ of patients with COVID-19 develop neurological symptoms: including headache, impaired consciousness and acute cerebrovascular disease. ${ }^{34}$ SARS-CoV-2 has neurotropic properties and has been found in cerebrospinal fluid (CSF) and brain tissue on autopsy. ${ }^{35}$

There is an increased risk of development of cerebrovascular events in patients with COVID-19. ${ }^{34}$ COVID-19 infection results in cytokine storm syndrome, which alongside coagulation dysfunction (resulting in abnormal D-dimer and platelet levels), increases the risk of cerebrobasilar disease. ${ }^{36}$ Therefore, patients at an increased risk of cerebrovascular disease should be informed of their risk from SARS-CoV-2 and monitored during a COVID-19 infection.

Viruses are a well-recognised cause of encephalitis, hence raising the possibility that SARS-CoV-2 can induce encephalitis in patients with COVID-19. Case reports from Wuhan describe classical signs of encephalitis in context of a typical COVID-19 presentation, without any evidence of other bacterial or viral infection. ${ }^{37}$ However, there may be difficulty in diagnosing SARS-CoV-2-induced encephalitis due to the dissemination of the virus throughout the CSF resulting in a low CSF viral titre. There have also been reports of acute haemorrhagic necrotising encephalopathy induced by the SARS-CoV-2 virus. ${ }^{38}$ Clinicians should be aware of patients who are SARS-CoV-2 positive who present with focal neurological signs and be prepared to treat as viral encephalitis.

There are several reports of Guillain-Barré syndrome (GBS) associated with SARS-CoV-2 infection from both China and Italy. ${ }^{39} 40$ The onset of symptoms was 5-10 days after onset of viral symptoms, which is a time period similar to other infections which cause GBS. ${ }^{41}$ Clinicians should be aware of the possibility of GBS caused by SARS-CoV-2 infection and give appropriate treatment.

\section{Coincidental finding of infection}

Due to the incubation period and nosocomial spread of the virus, COVID-19 must be considered as a potential causative agent in new diagnoses of hospital-acquired pneumonia during this pandemic. Patients admitted to hospital for other causes may become positive for SARS-CoV-2 infection during their stay, and therefore all patients must be regularly monitored for development of symptoms.

During the early phases of the pandemic, incidental findings of typical COVID-19 infection were found in imaging studies undertaken for other indications. For example, positron emission tomography-CT undertaken for standard oncological indications in a high prevalence region of Italy found a high number of incidental interstitial pneumonias consistent with COVID$19{ }^{42}$ Similar findings of incidental pneumonic changes consistent with COVID-19 were found in our own centre with trauma CT series undertaken for road traffic accidents and other injuries in previously asymptomatic patients.

\section{DIAGNOSTIC UNCERTAINTY}

Due to the rapid case transmission within the pandemic setting, any new cases of acute-onset respiratory illness may be treated as COVID-19 as default. Given the $75 \%$ sensitivity of RT-PCR detection and therefore presence of false negatives, ${ }^{7}$ in our hospital we adopted an edict that 'if it looks like COVID-19, it probably is COVID-19, so treat it as COVID-19'. This approach was adopted primarily to protect staff and allow for appropriate isolation precautions to be adopted in all suspected patients. However, as we continue into the later stages of the pandemic, this approach may lead to availability bias and thus misdiagnosis of COVID-19 in patients without the virus.

Availability bias is the tendency to judge diagnoses as more or less likely depending on how easily they come to mind, which is often influenced by recent patients seen. ${ }^{4344}$ In the context of the COVID-19 pandemic, patients fitting the typical case presentation but without a SARS-CoV-2 infection may be misdiagnosed due to this availability bias. This may lead to delayed treatment of the true aetiology, and thus an increased risk of adverse outcomes. Throughout this pandemic situation and despite the varied presentations of COVID-19, it is important that availability bias does not blind clinicians and lead to diagnostic oversight of other non-COVID-19 conditions during this time.

Potential issues related to this diagnostic uncertainty include: misdiagnosis of exacerbations of asthma and chronic obstructive pulmonary disease and delay in treatment with oral prednisolone; community-acquired pneumonia not caused by SARS-CoV-2 being misdiagnosed and patient subsequently 
Case vignette from our hospital

A 54-year-old man was admitted with a 2-week history of dyspnoea, followed by a 4-day history of myalgia, lethargy and an intermittent fever. The patient had a history of diabetes and asthma, and was a regular cigarette smoker. On examination he had a temperature (T) of $37^{\circ} \mathrm{C}$, blood pressure (BP) of $125 / 90 \mathrm{~mm}$ $\mathrm{Hg}$, heart rate of 109 beats per minute, respiratory rate (RR) of 29 breaths per minute and 02 saturations of $95 \%$ on air. On respiratory examination, there were bibasal crackles. The patient was admitted with an impression of COVID-19 infection and associated hypoxia. A chest X-ray was ordered and reported subtle multifocal air space opacification that was non-specific but could be consistent with viral pneumonitis. Importantly, he tested negative for SARS-CoV-2 on RT-PCR. The patient became unwell overnight, and was diagnosed with sepsis. A blood culture was obtained, and the patient started on intravenous amoxicillin. The following day, the patient's condition remained unchanged. SARS-CoV-2 RT-PCR testing was repeated and again was reported as negative. The patient underwent a chest CT scan that reported diffuse ground glass opacity affecting all lobes, with relative sparing of the peripheral parenchyma. The radiologist reported that these appearances were mostly in keeping with pneumocystis pneumonia (PCP) infection. Due to the possible diagnosis of PCP, the patient was tested for HIV infection. This returned as positive for HIV, with a viral load of $92742 \mathrm{c} / \mathrm{mL}$ and a CD4 count of $26 \times 10^{\wedge} 6 / \mathrm{L}$. His blood sample tested positive for PCP PCR.

\section{Pneumocystis pneumonia}

PCP is caused by Pneumocystis jirovecii, and results in a progressive dyspnoea mainly in immunocompromised individuals. In a mild presentation, patients report exertional dyspnoea. Typically, symptoms gradually worsen over time. In severe cases, patients may develop severe hypoxaemia and ARDS with a fatal outcome. PCP is an AIDS-defining illness, thus infected patients must be tested for HIV.

placed on a 'positive' ward; misdiagnosis of dyspnoea caused by pulmonary oedema secondary to heart failure and delay in diuretic treatment. Therefore, it is imperative that clinicians do not ignore their normal diagnostic processes and continue to consider a variety of differential diagnosis during this pandemic.

\section{Differential diagnoses and mimic conditions}

As seen in the case vignette above, early symptoms of COVID-19 such as fever, cough and dyspnoea are non-specific and thus can be associated with a variety of different conditions. Any acute viral infection will present in a similar way, which may result in diagnostic uncertainty. A differential diagnosis should include the possibility of a wide range of infectious and non-infectious disorders (figure 2).

\section{Respiratory mimics}

During this pandemic period, the majority of patients who present with symptoms fitting the case definition will be treated as suspected COVID-19. Therefore, it is important that rapid antigen detection for SARS-CoV-2 is performed, alongside other investigations to rule out common respiratory pathogens and non-infectious conditions. ${ }^{45}$

It is also important to remember that despite the high case load of COVID-19 in hospitals, at a similar time in previous years there will have been admissions with similar presentations, caused by other respiratory viruses, for example, influenza $\mathrm{A} / \mathrm{B} .{ }^{46}$ Presentation with these viruses will continue throughout this pandemic and clinicians must continue to correctly diagnose and treat these patients. Current guidance suggests that for hospitalised patients with an acute respiratory infection, there is no need to screen for influenza at the same time as SARS-CoV-2 testing. However, influenza testing should be considered where SARS-CoV-2 is negative, in severe infections and immunocompromised patients and in other cases where it is relevant for clinical management.

\section{Sepsis mimics}

Infection with SARS-CoV-2 can lead to sepsis. ${ }^{3}$ However, in patients with sepsis, consideration of other causes should not be forgotten. Pancultures (urine, sputum, blood) are essential in all patients with possible sepsis to rule out bacterial causes of sepsis. The Quick Sequential Organ Failure Assessment Score identifies patients with a possible sepsis diagnosis using three criteria: ${ }^{47}$

1. Low BP (systolic BP $\leq 100 \mathrm{~mm} \mathrm{Hg}$ ).

2. High RR ( $\geq 22$ breaths per minute).

3. Altered mentation (Glasgow Coma Scale $<15$ ).

Many patients with moderate to severe COVID-19 will meet at least two of these criteria, thus making the diagnosis of possible sepsis relatively easy. The real challenge is distinguishing COVID-19 viral sepsis from other cases of sepsis, which is often impossible in the early stages.

\section{INFECTIOUS CAUSES}

- Adenovirus

- Influenza

- Human metapneumovirus (HmPV)

- Parainfluenza

- Respiratory syncytial virus (RSV)

- Rhinovirus (common cold)

- Miliary Tuberculosis

- Cytomegalovirus

- Herpes Simplex And Hantavirus

- Pneumocystis Jirovecii

- Strongyloidosis

OTHER NON-INFECTIOUS CAUSES

\section{MALIGNANCY}

- Acute interstitial pneumonia

- Cryptogenic organising pneumonia

- Acute eosinophilic pneumonia
- Bronchoalveolar cell carcinoma

- Lymphangitis

- Acute leukaemia and lymphoma

Figure 2 Possible differential diagnosis of COVID-19. 
There is growing interest in the possible role of procalcitonin in distinguishing between viral and bacterial infections. Procalcitonin is a component of the innate proinflammatory response, which has been shown to be able to differentiate between bacterial infection and other abacterial inflammatory processes. ${ }^{48}$ However, evidence on the relevance of its role is mixed. Some studies suggest procalcitonin levels are lower in viral than bacterial infections. ${ }^{49}$ However, in a recent meta-analysis of 12 studies in 2408 patients with community-acquired pneumonia of known aetiology, the sensitivity and specificity of serum procalcitonin to differentiate between bacterial and non-bacterial pneumonia were both $55 \% .^{50}$ This suggests that a procalcitonin level alone is unlikely to provide enough evidence to differentiate between a viral and a bacterial infection. Current guidance by the Infectious Diseases Society of America does not recommend using procalcitonin as part of clinical judgement. ${ }^{51}$

Signs and symptoms commonly seen in sepsis can also be seen in non-infectious diseases, known as infectious mimicry. The cytokine storm seen in SARS-CoV-2 infection is due to HLH. Interestingly, a wide variety of non-infectious causes can also lead to HLH, including rheumatological and neoplastic causes. ${ }^{5}$ This infectious mimicry means that the infectious workup usually performed in sepsis does not always reveal the primary disease process. Therefore, clinicians should be aware of possible noninfectious mimics of COVID-19.

\section{Secondary bacterial infection}

To add to the difficulty of distinguishing sepsis due to a viral cause from other causes, coexistent infections are not uncommon. SARS-CoV-2 infection is known to cause a dysregulated immune response, with lymphopenia being a common feature, indicating a probable immune suppression stage. ${ }^{52}$ Therefore, in a manner similar to other respiratory viral infections, patients with COVID-19 are at high risk of developing secondary bacterial infections. These secondary infections must be identified and appropriately managed.

The prospect of another condition appearing alongside COVID-19 has been described as a 'medical chameleon', defined as when a condition is overlooked as it blends in with background disease. ${ }^{53}$ The background disease in this instance is COVID-19. For example, if a patient who was known positive for SARS-COV-2 infection developed sepsis, it would be understandable if the medical team attributed the cause to the virus and did not investigate further. However, if the patient had an undiagnosed coexistent bacterial infection contributing to their sepsis, the opportunity to treat with antibiotics would have been missed. Clearly this would lead to deterioration and an increased risk of adverse events.

\section{DISCUSSION}

Clinicians collect, process and interpret patient information to form a diagnosis and develop a management plan. The dualprocess model of clinical reasoning was hypothesised by Dr Pat Croskerry, and outlines the processes by which clinicians reach a diagnosis. This model has been adapted to fit a diagnosis of COVID-19 for this article (figure 3). ${ }^{54}$

When analysing all available information from a patient, clinicians use pattern processing; they may or may not recognise the disease pattern. If recognised, type 1 (intuitive) processes rapidly lead to a provisional diagnosis. However, if the pattern is not recognised, type 2 (rational or analytical) processes are used. This involves slower, deliberate hypothesis based on deductive clinical reasoning to form a diagnosis.

In COVID-19, a typical pattern of fever, cough and myalgia would fit the case definition and many clinicians would immediately form the provisional diagnosis of a SARS-CoV-2 infection via type 1 thought processes. However, the atypical presentations discussed in this article would result in a type 2 thought process, in order to distinguish between an atypical presentation of COVID-19 or a completely separate disease by full consideration of history, examination and investigation results.

As discussed, many conditions may mimic COVID-19 and therefore diagnostic errors could be made if clinicians rely fully on the immediate type 1 processes of pattern recognition. Dysrationalia over-ride is a switch from type 1 to type 2 thinking, when a presentation results in deliberation as to whether this is truly COVID-19 or another mimic disease. Executive over-ride occurs when type 2 analysis leads to consideration of whether

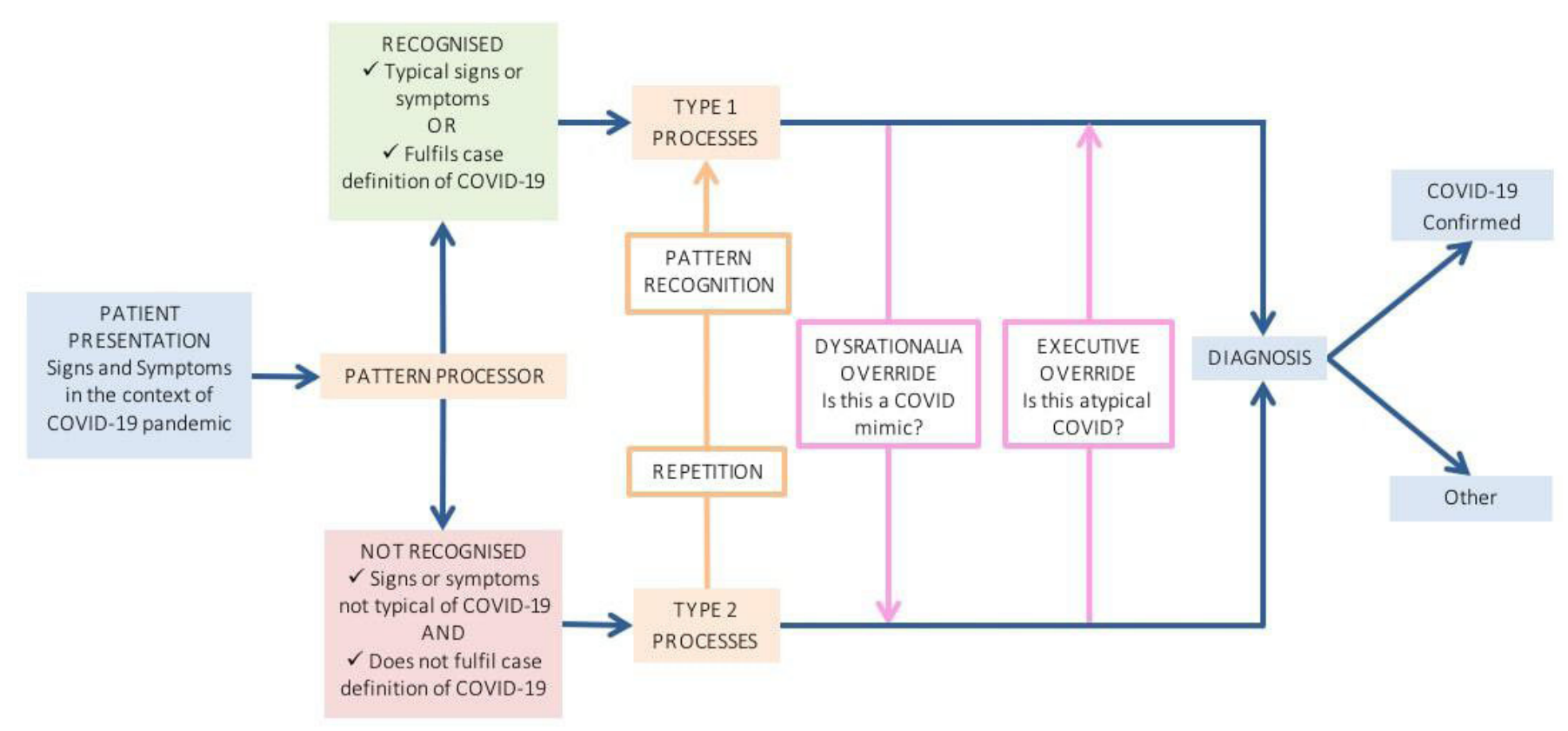

Figure 3 Dual-process model of clinical reasoning ${ }^{54}$ adapted to COVID-19. 
Main messages

- COVID-19 presents to acute care as a viral pneumonia, and is easily identified by pattern recognition of the typical respiratory symptoms.

- A number of atypical presentations affecting a variety of organ systems have become apparent, including anosmia, diarrhoea and neurological features.

- Diagnostic reasoning using intuitive processes can rapidly identify typical COVID-19 cases in a pandemic situation, but alternative cognitive processes are required to consider other COVID-19 mimic diagnoses.

\section{Current research questions}

- How are the usual diagnostic reasoning processes affected during a pandemic situation such as COVID-19?

- To what extent is the diagnosis of COVID-19 missed as a result of atypical presentations?

- What impact does the treatment delay or omission in patients misdiagnosed with COVID-19 have on morbidity and mortality of the true diagnosis?

\section{What is already known on the subject}

- Many disease processes have both atypical features and mimics that complicate diagnosis

- Clinicians use Type 1 and Type 2 processes for diagnostic reasoning

- COVID-19 is an emerging viral infection caused by SARSCoV-2 that has a wide range of presentations

this may be an atypical presentation of COVID-19. Over time, repetition of atypical presentations (such as anosmia) may lead to pattern recognition and switching from a type 2 to a type 1 process and rapid diagnosis of COVID-19 infection.

\section{CONCLUSION}

While SARS-CoV-2 is likely to remain on the differential diagnostic list for a plethora of presentations for the foreseeable future, clinicians should be cautious of ignoring other potential diagnoses. An awareness of atypical presentations allows SARS-CoV-2 to be a differential so that it can be appropriately investigated. A knowledge of infectious mimics prevents COVID-19 from overshadowing other diagnoses, hence preventing delayed diagnosis or even misdiagnosis and consequent adverse outcomes for patients.

In the postpandemic period, it is important to remember that a patient presenting with apparent COVID-19 infection may or may not have COVID-19. Clinicians must continue to investigate fully and treat appropriately, as making assumptions can significantly hinder patient outcomes.

\section{Twitter Jamie J Coleman @colemajj}

Contributors JJC, EJM, AHB and ES undertook the initial drafting of the manuscript. EJM and AHB undertook literature searches. KM contributed the case vignettes. All authors contributed to the final manuscript and approved the final version.

Funding The authors have not declared a specific grant for this research from any funding agency in the public, commercial or not-for-profit sectors.
Disclaimer The views expressed in this publication are not necessarily those of the Department of Health, University Hospital Birmingham or the University of Birmingham.

Competing interests None declared.

Patient consent for publication Not required.

Provenance and peer review Not commissioned; internally peer reviewed.

Data availability statement No data are available. Not applicable.

Open access This is an open access article distributed in accordance with the Creative Commons Attribution Non Commercial (CC BY-NC 4.0) license, which permits others to distribute, remix, adapt, build upon this work non-commercially, and license their derivative works on different terms, provided the original work is properly cited, appropriate credit is given, any changes made indicated, and the use is non-commercial. See: http://creativecommons.org/licenses/by-nc/4.0/.

\section{ORCID iD}

Jamie J Coleman http://orcid.org/0000-0002-7512-5153

\section{REFERENCES}

1 World Health Organisation. Coronavirus disease (COVID-19) pandemic, 2020. Available: https://www.who.int/emergencies/diseases/novel-coronavirus-2019 [Accessed 20 Mar 2020].

2 Zhou F, Yu T, Du R, et al. Clinical course and risk factors for mortality of adult inpatients with COVID-19 in Wuhan, China: a retrospective cohort study. Lancet 2020;395:1054-62.

3 Mason RJ. Pathogenesis of COVID-19 from a cell biology perspective. Eur Respir J 2020;55:2000607.

4 Peluso MJ, Chia D, Sheen W, et al. Infectious mimicry complicates diagnosis in hemophagocytic syndrome caused by anaplastic large-cell lymphoma. Case Rep Med 2012;2012:1-6.

5 Public Health England. COVID-19: investigation and initial clinical management of possible cases, 2020. Available: https://www.gov.uk/government/publications/wuhannovel-coronavirus-initial-investigation-of-possible-cases/investigation-and-initialclinical-management-of-possible-cases-of-wuhan-novel-coronavirus-wn-cov-infection [Accessed 18 Apr 2020].

6 Guan W-J, Ni Z-Y, Hu Y, et al. Clinical characteristics of coronavirus disease 2019 in China. N Engl J Med 2020;382:1708-20.

7 Wölfel R, Corman VM, Guggemos W, et al. Virological assessment of hospitalized patients with COVID-2019. Nature 2020;382. doi:10.1038/s41586-020-2196-X. [Epub ahead of print: 01 Apr 2020].

8 Wu JT, Leung K, Bushman M, et al. Estimating clinical severity of COVID-19 from the transmission dynamics in Wuhan, China. Nat Med 2020;26:506-10.

9 Wu C, Chen X, Cai Y, et al. Risk factors associated with acute respiratory distress syndrome and death in patients with coronavirus disease 2019 pneumonia in Wuhan, China. JAMA Intern Med 2020. doi:10.1001/jamainternmed.2020.0994. [Epub ahead of print: 13 Mar 2020].

10 El Chakhtoura NG, Bonomo RA, Jump RLP. Influence of aging and environment on presentation of infection in older adults. Infect Dis Clin North Am 2017;31:593-608.

11 Xie J, Tong Z, Guan X, et al. Critical care crisis and some recommendations during the COVID-19 epidemic in China. Intensive Care Med 2020;22. doi:10.1007/s00134-02005979-7. [Epub ahead of print: 02 Mar 2020].

12 British Geriatrics Society, European Delirium Association, Old Age Psychiatry Faculty (Royal College of Psychiatrists). Coronavirus: managing delirium in confirmed and suspected cases, 2020. Available: https://www.bgs.org.uk/resources/coronavirusmanaging-delirium-in-confirmed-and-suspected-cases [Accessed $20 \mathrm{Apr} 2020$ ].

13 Giacomelli A, Pezzati L, Conti F, et al. Self-Reported olfactory and taste disorders in patients with severe acute respiratory coronavirus 2 infection: a cross-sectional study. Clin Infect Dis 2020;395.

14 Xydakis MS, Dehgani-Mobaraki P, Holbrook EH, et al. Smell and taste dysfunction in patients with COVID-19. Lancet Infect Dis 2020. doi:10.1016/S1473-3099(20)302930. [Epub ahead of print: 15 Apr 2020].

15 Hopkins C, Kumar N. Loss of sense of smell as marker of COVID-19 infection, 2020. Available: https://www.entuk.org/loss-sense-smell-marker-covid-19-infection [Accessed 21 Apr 2020].

16 Desforges M, Le Coupanec A, É B, et al. Neuroinvasive and neurotropic human respiratory coronaviruses: potential neurovirulent agents in humans. Springer India, 2014: 75-96.

17 YC L, Bai WZ, Hashikawa T. The Neuroinvasive potential of SARS-CoV2 may play a role in the respiratory failure of COVID-19 patients. J Med Virol 2020;92.

18 Zheng Y-Y, Ma Y-T, Zhang J-Y, et al. COVID-19 and the cardiovascular system. Nat Rev Cardiol 2020;17:259-60.

19 Clerkin KJ, Fried JA, Raikhelkar J, et al. Coronavirus disease 2019 (COVID-19) and cardiovascular disease. Circulation 2020;58.

20 Liu PP, Blet A, Smyth D, et al. The science underlying COVID-19: implications for the cardiovascular system. Circulation 2020;382. doi:10.1161/ CIRCULATIONAHA.120.047549. [Epub ahead of print: 15 Apr 2020]. 
$21 \mathrm{Hu} \mathrm{H}$, Ma F, Wei X, et al. Coronavirus fulminant myocarditis treated with glucocorticoid and human immunoglobulin. Eur Heart J 2020.

22 Madjid M, Safavi-Naeini P, Solomon SD, et al. Potential effects of coronaviruses on the cardiovascular system. JAMA Cardiology 2020.

23 Tapé C, Byrd KM, Aung S, et al. COVID-19 in a patient presenting with syncope and a normal chest X-ray. R I Med J 2020;103:50-1.

24 Holshue ML, DeBolt C, Lindquist S, et al. First case of 2019 novel coronavirus in the United States. N Engl J Med 2020;382:929-36.

25 Wan Y, Li J, Shen L, et al. Enteric involvement in hospitalised patients with COVID-19 outside Wuhan. Lancet Gastroenterol Hepatol 2020. doi:10.1016/S24681253(20)30118-7. [Epub ahead of print: 15 Apr 2020].

26 Zhang W, Du R-H, Li B, et al. Molecular and serological investigation of 2019-nCoV infected patients: implication of multiple shedding routes. Emerg Microbes Infect 2020;9:386-9.

27 Huang C, Wang Y, Li X, et al. Clinical features of patients infected with 2019 novel coronavirus in Wuhan, China. The Lancet 2020;395:497-506.

28 Chen N, Zhou M, Dong X, et al. Epidemiological and clinical characteristics of 99 cases of 2019 novel coronavirus pneumonia in Wuhan, China: a descriptive study. Lancet 2020;395:507-13.

29 Wang D, Hu B, Hu C, et al. Clinical characteristics of 138 hospitalized patients with 2019 novel coronavirus-infected pneumonia in Wuhan, China. JAMA 2020;323:1061-9.

30 Shi H, Han X, Jiang N, et al. Radiological findings from 81 patients with COVID-19 pneumonia in Wuhan, China: a descriptive study. Lancet Infect Dis 2020;20:425-34.

$31 \mathrm{Xu}$ X-W, Wu X-X, Jiang X-G, et al. Clinical findings in a group of patients infected with the 2019 novel coronavirus (SARS-Cov-2) outside of Wuhan, China: retrospective case series. BMJ 2020;368:m606.

32 Zhang C, Shi L, Wang F-S. Liver injury in COVID-19: management and challenges. Lancet Gastroenterol Hepatol 2020;5:428-30.

33 Bangash MN, Patel J, Parekh D. COVID-19 and the liver: little cause for concern. Lancet Gastroenterol Hepatol 2020. doi:10.1016/\$2468-1253(20)30084-4. [Epub ahead of print: 20 Mar 2020].

34 Mao L, Wang M, Chen S, et al. Neurological manifestations of hospitalized patients with COVID-19 in Wuhan. China: A Retrospective Case Series Study, 2020.

$35 \mathrm{Xu} \mathrm{Z}$, Shi L, Wang Y, et al. Pathological findings of COVID-19 associated with acute respiratory distress syndrome. Lancet Respir Med 2020;8:420-2.

36 Wu Y, Xu X, Chen Z, et al. Nervous system involvement after infection with COVID-19 and other coronaviruses. Brain Behav Immun 2020. doi:10.1016/j.bbi.2020.03.031. [Epub ahead of print: 30 Mar 2020].

37 Ye M, Ren Y, Lv T. Encephalitis as a clinical manifestation of COVID-19. Brain Behav Immun 2020. doi:10.1016/j.bbi.2020.04.017. [Epub ahead of print: 10 Apr 2020].
38 Poyiadji N, Shahin G, Noujaim D, et al. COVID-19-associated acute hemorrhagic necrotizing encephalopathy: CT and MRI features. Radiology 2020

39 Zhao $H$, Shen D, Zhou H, et al. Guillain-Barré syndrome associated with SARS-CoV-2 infection: causality or coincidence? The Lancet Neurology 2020;19:383-4.

40 Toscano G, Palmerini F, Ravaglia S, et al. Guillain-Barré syndrome associated with SARS-CoV-2. New England Journal of Medicine 2020.

41 Wijdicks EFM, Klein CJ, Syndrome G-B. Guillain-Barré syndrome. Mayo Clin Proc 2017;92:467-79.

42 Albano D, Bertagna F, Bertoli M, et al. Incidental findings suggestive of COVID-19 in asymptomatic patients undergoing nuclear medicine procedures in a high-prevalence region. J Nucl Med 2020;61:632-6.

43 Croskerry $\mathrm{P}$. The importance of cognitive errors in diagnosis and strategies to minimize them. Acad Med 2003;78:775-80.

44 Ely JW, Graber ML, Croskerry P. Checklists to reduce diagnostic errors. Acad Med 2011;86:307-13.

45 Cascella M, Rajnik M, Cuomo A, et al. Features, evaluation and treatment coronavirus (COVID-19). StatPearls Publishing LLC, 2020.

46 Public Health England. Weekly national flu reports: 2018 to 2019 season, 2019. Available: https://www.gov.uk/government/statistics/weekly-national-flu-reports2018-to-2019-season [Accessed 21 Apr 2020].

47 Singer M, Deutschman CS, Seymour CW, et al. The third International consensus definitions for sepsis and septic shock (Sepsis-3). JAMA 2016;315:801.

48 Delèvaux I, André $M$, Colombier $M$, et al. Can procalcitonin measurement help in differentiating between bacterial infection and other kinds of inflammatory processes? Ann Rheum Dis 2003;62:337-40.

49 Self WH, Balk RA, Grijalva CG, et al. Procalcitonin as a marker of etiology in adults hospitalized with community-acquired pneumonia. Clin Infect Dis 2017;65:183-90.

50 Kamat IS, Ramachandran V, Eswaran H, et al. Procalcitonin to distinguish viral from bacterial pneumonia: a systematic review and meta-analysis. Clin Infect Dis 2019.

51 Kalil AC, Metersky ML, Klompas M, et al. Management of adults with hospitalacquired and ventilator-associated pneumonia: 2016 clinical practice guidelines by the infectious diseases Society of America and the American thoracic Society. Clin Infect Dis 2016:63:e61-111.

52 Li H, Liu L, Zhang D, et al. SARS-CoV-2 and viral sepsis: observations and hypotheses. Lancet 2020. doi:10.1016/S0140-6736(20)30920-X. [Epub ahead of print: $17 \mathrm{Apr}$ 2020].

53 Nickel CH, Bingisser R. Mimics and chameleons of COVID-19. Swiss Med Wkly 2020;150:w20231.

54 Croskerry P. A universal model of diagnostic reasoning. Acad Med 2009;84:1022-8. 